Research Article

\title{
Computing 2-twinless blocks
}

\author{
Raed Jaberi*
}

Department of Software and Information Systems, Tishreen University, Lattakia, Syria

(Received: 22 June 2020. Received in revised form: ${ }^{\dagger} 30$ January 2021. Accepted: 16 February 2021. Published online: 20 February 2021.)

(c) 2021 the author. This is an open access article under the CC BY (International 4.0) license (www.creativecommons.org/licenses/by/4.0/)

\begin{abstract}
Let $G=(V, E)$ be a directed graph. A 2-twinless block in $G$ is a maximal subset $B \subseteq V$ of size at least 2 such that for every pair of distinct vertices $x, y \in B$, and for every vertex $w \in V \backslash\{x, y\}$, the vertices $x, y$ are in the same twinless strongly connected component of $G \backslash\{w\}$. In this paper, algorithms for computing the 2-twinless blocks of a directed graph are presented.
\end{abstract}

Keywords: directed graphs; connectivity; graph algorithms; 2-blocks; twinless strongly connected graphs.

2020 Mathematics Subject Classification: 05C85, 05C20.

\section{Introduction}

Let $G=(V, E)$ be a directed graph. The graph $G$ is twinless strongly connected if it contains a strongly connected spanning subgraph $\left(V, E^{t}\right)$ such that $E^{t}$ does not contain any pair of antiparallel edges. A twinless strongly connected component of $G$ is a maximal subset $C_{t} \subseteq V$ such that the induced subgraph on $C_{t}$ is twinless strongly connected. A strong articulation point of $G$ is a vertex whose removal increases the number of strongly connected components of $G$. A strong bridge of $G$ is an edge whose deletion increases the number of strongly connected components of $G$. A strongly connected graph is 2-vertex-connected if it has at least 3 vertices and it has no strong articulation points. A 2-vertex-connected component of $G$ is a maximal vertex subset $C^{v} \subseteq V$ such that the induced subgraph on $C^{v}$ is 2-vertex-connected. A 2-directed block in $G$ is a maximal vertex subset $B^{d} \subseteq V$ with $\left|B^{d}\right|>1$ such that for any distinct vertices $x, y \in B^{d}$, the graph $G$ contains two vertex-disjoint paths from $x$ to $y$ and two vertex-disjoint paths from $y$ to $x$. A 2-edge block in $G$ is a maximal subset $B^{e b} \subseteq V$ with $\left|B^{e b}\right|>1$ such that for any distinct vertices $v, w \in B^{e b}$, there are two edge-disjoint paths from $v$ to $w$ and two edge-disjoint paths from $w$ to $v$ in $G$. A 2-strong block in $G$ is a maximal vertex subset $B^{s} \subseteq V$ with $\left|B^{s}\right|>1$ such that for each pair of distinct vertices $x, y \in B^{s}$ and for every vertex $u \in V \backslash\{x, y\}$, the vertices $x$ and $y$ are in the same strongly connected component of the graph $G \backslash\{u\}$. A twinless articulation point of $G$ is a vertex whose removal increases the number of twinless strongly connected components of $G$. A 2-twinless block in $G$ is a maximal vertex set $B \subseteq V$ of size at least 2 such that for each pair of distinct vertices $x, y \in B$, and for each vertex $w \in V \backslash\{x, y\}$, the vertices $x, y$ are in the same twinless strongly connected component of $G \backslash\{w\}$. Notice that 2-strong blocks are not necessarily 2-twinless blocks (see Figure 1).

A twinless strongly connected graph $G$ is said to be 2-vertex-twinless-connected if it has at least three vertices and it does not contain any twinless articulation point [19]. A 2-vertex-twinless-connected component is a maximal subset $U^{2 v t} \subseteq V$ such that the induced subgraph on $U^{2 v t}$ is 2-vertex-twinless-connected. While 2-vertex-twinless-connected components have at least linear number of edges, the subgraphs induced by 2-twinless blocks do not necessarily contain edges.

Strongly connected components can be found in linear time [25]. In 2006, Raghavan [22] showed that the twinless strongly connected component of a directed graph can be found in linear time. In 2010, Georgiadis [7] presented an algorithm to check whether a strongly connected graph is 2-vertex-connected in linear time. Italiano et al. [15] gave linear time algorithms for identifying all the strong articulation points and strong bridges of a directed graph. Their algorithms are based on dominators [1-4,6,21]. In 2014, Jaberi [17] presented algorithms for computing the 2-vertex-connected components of directed graphs in $O(\mathrm{~nm})$ time (published in [16]). Henzinger et al. [14] gave algorithms for calculating the 2-vertex-connected components in $O\left(n^{2}\right)$ time. Jaberi [18] presented algorithms for computing 2-blocks in directed graphs. Georgiadis et al. [9,10] gave linear time algorithms for determining 2-edge blocks. Georgiadis et al. [11,12] also gave linear time algorithms for calculating 2-directed blocks and 2-strong blocks. Georgiadis et al. [13] and Luigi et al. [20] performed 


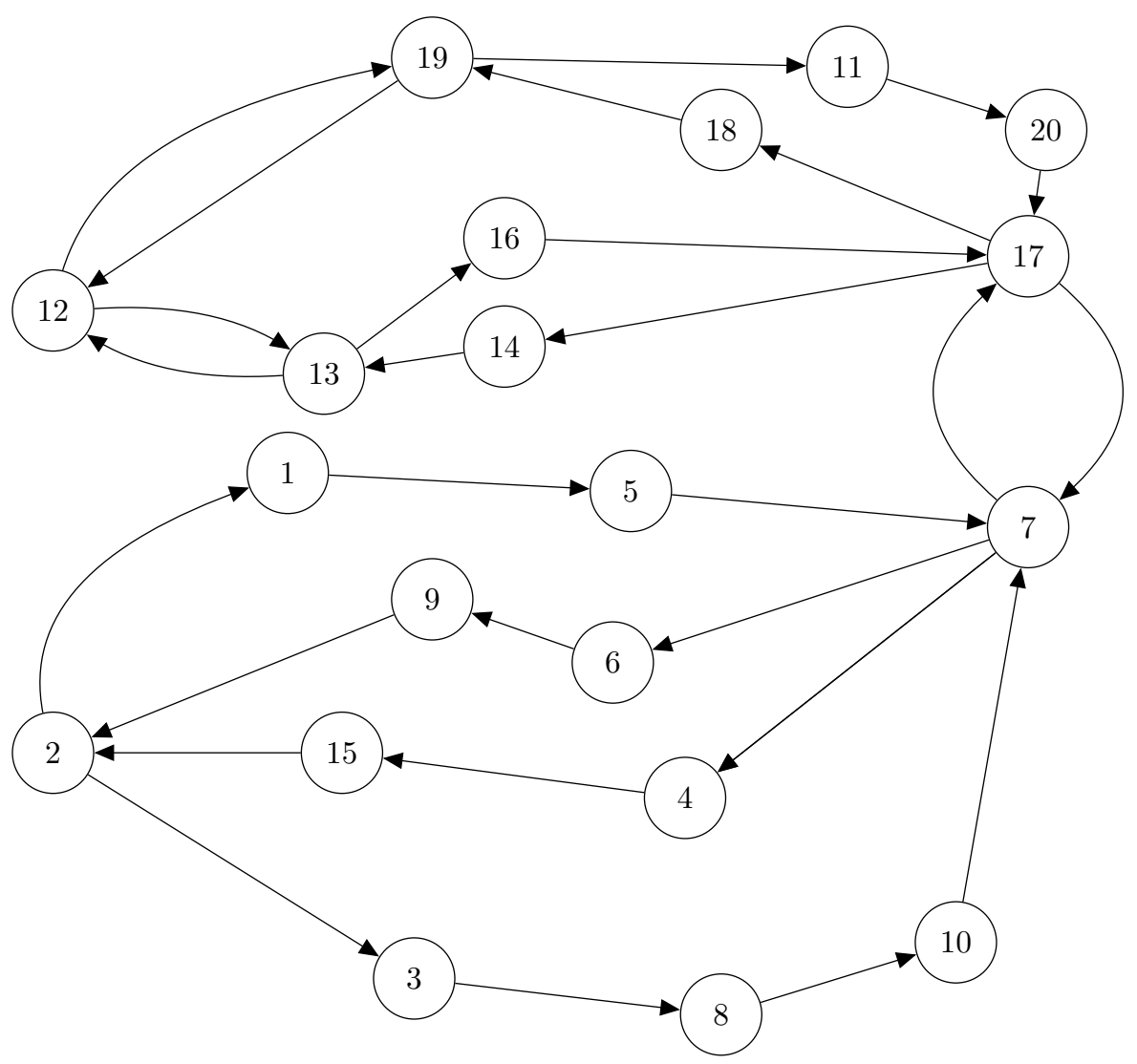

Figure 1: A strongly connected graph $G$, which contains two 2-strong blocks $C_{1}=\{2,7\}, C_{2}=\{12,13,17,19\}$, and one 2 -twinless block $B=\{2,7\}$. Notice that the vertices 12 and 17 do not belong to the same twinless strongly connected component of $G \backslash\{13\}$.

experimental studies of recent algorithms that calculate 2-blocks and 2-connected components in directed graphs. In 2019, Jaberi [19] presented an algorithm for computing 2-vertex-twinless-connected components. Georgiadis and Kosinas [8] gave a linear time algorithm for calculating twinless articulation points.

In the next section, we show that the 2-twinless blocks of a directed graph can be calculated in $O\left(n^{3}\right)$ time.

\section{Algorithm for computing 2-twinless blocks}

In this section we present an algorithm for computing the 2-twinless blocks of a twinless strongly connected graph. Since twinless strongly connected components do not share vertices of the same 2-twinless block, we consider only twinless strongly connected graphs. Let $G=(V, E)$ be a twinless strongly connected graph. We define a relation $\stackrel{2 t}{\rightsquigarrow}$ as follows. For any distinct vertices $x, y \in V$, we write $x \stackrel{2 t}{m} y$ if for all vertices $w \in V \backslash\{x, y\}$, the vertices $x, y$ are in the same twinless strongly connected component of $G \backslash\{w\}$. By definition, a 2-twinless block in $G$ is a maximal subset $B^{2 t} \subseteq V$ with $\left|B^{2 t}\right|>1$ such that for every two vertices $x, y \in B^{2 t}$, we have $x \stackrel{2 t}{m} y$.

The next lemma shows that 2-twinless blocks share at most one vertex.

Lemma 2.1. Let $G=(V, E)$ be a twinless strongly connected graph. Let $B_{1}^{2 t}, B_{2}^{2 t}$ be distinct 2-twinless blocks in $G$. Then $\left|B_{1}^{2 t} \cap B_{2}^{2 t}\right| \leq 1$.

Proof. Suppose for the sake of contradiction that $B_{1}^{2 t}$ and $B_{2}^{2 t}$ have at least two vertices in common. Clearly, $B_{1}^{2 t} \cup B_{2}^{2 t}$ is not a 2-twinless block in $G$. Let $x$ and $y$ be vertices belonging to $B_{1}^{2 t}$ and $B_{2}^{2 t}$, respectively, such that $x, y \notin B_{1}^{2 t} \cap B_{2}^{2 t}$. Let $z$ be any vertex in $V \backslash\{x, y\}$. Since $\left|B_{1}^{2 t} \cap B_{2}^{2 t}\right|>1$, there is a vertex $v$ in $\left(B_{1}^{2 t} \cap B_{2}^{2 t}\right) \backslash\{z\}$. Note that $x, v$ are in the same twinless strongly connected component of $G \backslash\{z\}$ since $x, v \in B_{1}^{2 t}$. Moreover, $v$ and $y$ lie in the same twinless strongly connected component of $G \backslash\{z\}$. By Lemma 1 of [22], $x$ and $y$ are in the same twinless strongly connected component of $G \backslash\{z\}$. Therefore, $x, y$ belong to the same 2-twinless block.

The following lemma shows an interesting property of the relation $2 t$

Lemma 2.2. Let $G=(V, E)$ be a twinless strongly connected graph and let $\left\{v_{0}, v_{1}, \ldots, v_{l}\right\}$ be set of vertices of $G$ such that $v_{l} \stackrel{2 t}{\leftrightarrow} v_{0}$ and $v_{i-1} \stackrel{2 t}{\rightsquigarrow} v_{i}$ for $i \in\{1,2 \ldots, l\}$. Then $\left\{v_{0}, v_{1}, \ldots, v_{l}\right\}$ is a subset of a 2-twinless block in $G$. 
Proof. Assume for the purpose of contradiction that there are two vertices $v_{r}$ and $v_{q}$ in $G$ such that $v_{r}$ and $v_{q}$ are in distinct 2-twinless blocks of $G$ and $r, q \in\{0,1, \ldots, l\}$. Suppose without loss of generality that $r<q$. Then there is a vertex $z \in V \backslash\left\{v_{r}, v_{q}\right\}$ such that $v_{r}$ and $v_{q}$ are in distinct twinless strongly connected components of $G \backslash\{z\}$. We distinguish two cases.

1. $z \in\left\{v_{r+1}, v_{r+2}, \ldots, v_{q-1}\right\}$. In this case, the vertices $v_{i-1}, v_{i}$ belong to the same twinless strongly connected component of $G \backslash\{z\}$ for each $i \in\{1,2, \ldots, r\} \cup\{q+1, q+2, \ldots, l\}$. Moreover, the vertices $v_{0}, v_{l}$ are in the same twinless strongly connected component of $G \backslash\{z\}$ because $v_{0} \stackrel{2 t}{m} v_{l}$. Therefore, the vertices $v_{r}, v_{q}$ are in the same twinless strongly connected component of the graph $G \backslash\{z\}$, a contradiction.

2. $z \notin\left\{v_{r+1}, v_{r+2}, \ldots, v_{q-1}\right\}$. Then, for each $i \in\{r+1, r+2, \ldots, q\}$, the vertices $v_{i-1}, v_{i}$ lie in the same twinless strongly connected component of $G \backslash\{z\}$. Consequently, the vertices $v_{r}, v_{q}$ belong to the same twinless strongly connected component of the graph $G \backslash\{z\}$, a contradiction.

Let $G=(V, E)$ be a twinless strongly connected graph. We construct the 2-twinless block graph $G^{2 t}=\left(V^{2 t}, E^{2 t}\right)$ of $G$ as follows. For every 2-twinlesss block $B_{i}$, we add a vertex $v_{i}$ to $V^{2 t}$. Moreover, for each vertex $v \in V$, if $v$ belongs to at least two distinct 2-twinless blocks, we add a vertex $v$ to $V^{2 t}$. For any distinct 2-twinless blocks $B_{i}, B_{j}$ with $B_{i} \cap B_{j}=\{v\}$, we put two undirected edges $\left(v_{i}, v\right),\left(v, v_{j}\right)$ into $E^{2 t}$.

Lemma 2.3. The 2-twinless block graph of a twinless strongly connected graph is a forest.

Proof. This result follows from Lemma 2.2 and Lemma 2.1.

Lemma 2.4. Let $G=(V, E)$ be a twinless strongly connected graph and let $x, y$ be distinct vertices in $G$. Suppose that $v \in V \backslash\{x, y\}$ is not a twinless articulation point. Then $x, y$ are in the same twinless strong connected component of $G \backslash\{v\}$.

Proof. Immediate from the definition.

Now, we give an algorithm for computing the 2-twinless blocks of a twinless strongly connected graph $G=(V, E)$.

\section{Algorithm 2.1.}

Input: A twinless strongly connected graph $G=(V, E)$.

Output: The 2-twinless blocks of $G$.

1 if $G$ is 2-vertex-twinless connected then

Output $V$.

else

Let $S$ be an $n \times n$ matrix.

Initialize $S$ with 1 s.

determine the twinless articulation points of $G$.

for each twinless articulation point $z$ of $G$ do

Identify the twinless strongly connected components of $G \backslash\{z\}$.

for each pair $(v, w) \in(V \backslash\{z\}) \times(V \backslash\{z\})$ do

if $v, w$ in different twinless strongly connected components of $G \backslash\{z\}$ then

$E^{b} \leftarrow \emptyset$. $S[v, w] \leftarrow 0$.

for each pair $(v, u) \in V \times V$ do

if $S[v, u]=1$ and $S[u, v]=1$ then

$E^{b} \leftarrow E^{b} \cup\{(v, u)\}$.

calculate the blocks of size $\geq 2$ of $G^{b}=\left(V, E^{b}\right)$ and output them.

The correctness of Algorithm 2.1 follows from the following lemma.

Lemma 2.5. A vertex subset $B \subseteq V$ is a 2-twinless block of $G$ if and only if $B$ is a block of the undirected graph $G^{b}=\left(V, E^{b}\right)$ which is constructed in lines $12-15$ of Algorithm 2.1

Proof. It follows from Lemma 2.2 and Lemma 2.4 .

Theorem 2.1. Algorithm 2.1 runs in $O\left(n^{3}\right)$ time. 
Proof. Georgiadis and Kosinas [8] showed that the twinless articulation points can be computed in linear time. The initialization of matrix $S$ takes $O\left(n^{2}\right)$ time. The number of iterations of the for-loop in lines 7-11 is at most $n$ because the number of twinless articulation points is at most $n$. Consequently, lines $7-11$ require $O\left(n^{3}\right)$. Furthermore, the blocks of an undirected graph can be found in linear time [24,25].

The following lemma shows an important property of $G^{b}$.

Lemma 2.6. The graph $G^{b}$ which is constructed in lines $12-15$ of Algorithm 2.1 is chordal.

Proof. It follows from Lemma 2.2 .

By Lemma 2.6, one can calculate the maximal cliques of $G^{b}$ instead of blocks. The maximal cliques of a chordal graph can be calculated in linear time [5,23].

\section{An improved version of Algorithm 2.1}

In this section, we present an improved version of Algorithm 2.1.

The following lemma shows a connection between 2-twinless blocks and 2-strong blocks.

Lemma 3.1. Let $G=(V, E)$ be a twinless strongly connected graph. Suppose that $B_{t}$ is a 2-twinless block in $G$. Then $B_{t}$ is a subset of a 2-strong block in $G$.

Proof. Let $v$ and $w$ be distinct vertices in $B_{t}$, and let $x \in V \backslash\{v, w\}$. By definition, the vertices $v, w$ belong to the same twinless strongly connected component $C$ of $G \backslash\{x\}$. Since $C$ is a subset of a strongly connected component of $G$, the vertices $v, w$ also lie in the same strongly connected component of $G \backslash\{x\}$. Consequently, $v, w$ are in the same 2-strong block in $G$.

The next algorithm describes an improved version of Algorithm 2.1 which is based on Lemma 3.1 and Lemma 2.2.

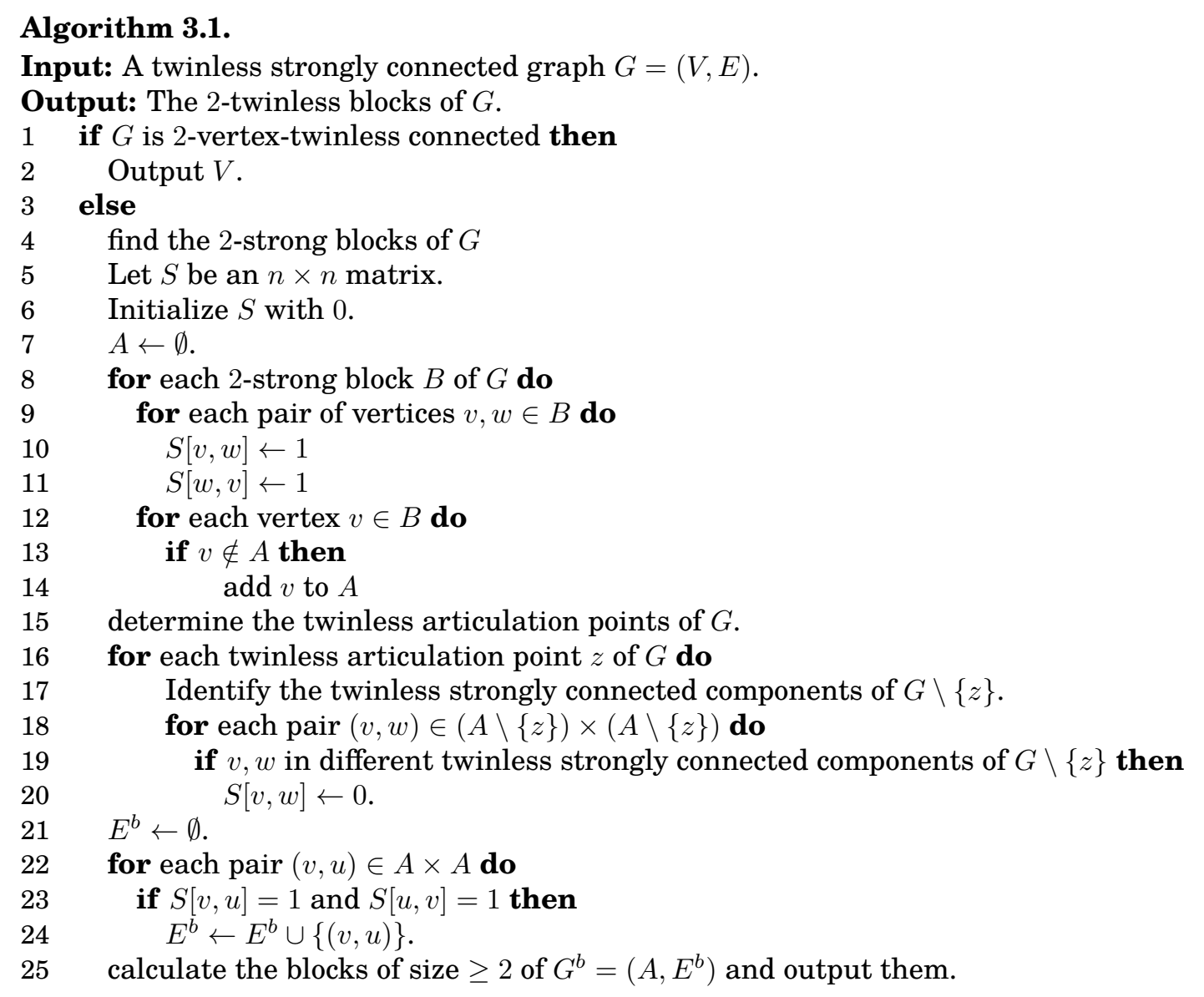

Theorem 3.1. The running time of Algorithm 3.1 is $O\left(t\left(s^{2}+m\right)+n^{2}\right)$, where $s=|A|$ and $t$ is the number of twinless articulation points of $G$. 
Proof. The 2-strong blocks of a directed graph can be computed in linear time [11]. Furthermore, the twinless articulation points of a directed graph can be identified in linear time using the algorithm of Georgiadis and Kosinas [8]. Since the number of iterations of the for-loop in lines $16-20$ is at most $t$, lines 16-20 take $O\left(t\left(s^{2}+m\right)\right)$ time.

Let $G=(V, E)$ be a twinless strongly connected graph. If the refine operation defined in $[11,20]$ is used to refine the 2-strong blocks of $G$ for all twinless articulation points, then the 2-twinless blocks of a directed graph $G=(V, E)$ can be computed in $O(t m)$ time, where $t$ is the number of twinless articulation points of $G$.

We leave as an open problem whether the 2-twinless blocks of a directed graph can be calculated in linear time.

\section{Acknowledgement}

The author would like to thank the anonymous reviewers for their helpful comments and suggestions.

\section{References}

[1] S. Alstrup, D. Harel, P.W. Lauridsen, M. Thorup, Dominators in linear time, SIAM J. Comput. 28 (1999) $2117-2132$.

[2] A. L. Buchsbaum, L. Georgiadis, H. Kaplan, A. Rogers, R. E. Tarjan, J. R. Westbrook, Linear-time algorithms for dominators and other pathevaluation problems, SIAM J. Comput. 38 (2008) 1533-1573.

[3] D. Firmani, G. F. Italiano, L. Laura, A. Orlandi, F. Santaroni, Computing strong articulation points and strong bridges in large scale graphs, In: R. Klasing (Ed.), Experimental Algorithms, SEA 2012, Lecture Notes in Computer Science, Vol. 7276, Springer, Berlin, 2012 , pp. $195-207$.

[4] D. Firmani, L. Georgiadis, G. F. Italiano, L. Laura, F. Santaroni, Strong articulation points and strong bridges in large scale graphs, Algorithmica 74 (2016) 1123-1147.

[5] F. Gavril, Algorithms for minimum coloring, maximum clique, minimum covering by cliques, and maximum independent set of a chordal graph, SIAM J. Comput. 1 (1972) 180-187.

[6] L. Georgiadis, R. E. Tarjan, R F. Werneck, Finding dominators in practice, J. Graph Algorithms Appl. 10 (2006) 69-94.

[7] L. Georgiadis, Testing 2-vertex connectivity and computing pairs of vertex-disjoint s-t paths in digraphs, In: S. Abramsky, C. Gavoille, C. Kirchner, F. Meyer auf der Heide, P. G. Spirakis (Eds.), Automata, Languages and Programming, ICALP 2010, Lecture Notes in Computer Science, Vol. 6198, Springer, Berlin, 2021, pp. 738-749.

[8] L. Georgiadis, E. Kosinas, Linear-time algorithms for computing twinless strong articulation points and related problems, Proceedings of the 31st International Symposium on Algorithms and Computation (ISAAC 2020), pp. 38:1-38:16.

[9] L. Georgiadis, G. F. Italiano, L. Laura, N. Parotsidis, 2-Edge connectivity in directed graphs, Proceedings of the 2015 Annual ACM-SIAM Symposium on Discrete Algorithms, 2015, pp. 1988-2005.

[10] L. Georgiadis, G. F. Italiano, L. Laura, N. Parotsidis, 2-Edge connectivity in directed graphs, ACM Trans. Algorithms 13 (2016) Art\# 9.

[11] L. Georgiadis, G. F. Italiano, L. Laura, N. Parotsidis, 2-Vertex connectivity in directed graphs, In: M. Halldórsson, K. Iwama, N. Kobayashi, B. Speckmann (Eds.), Automata, Languages, and Programming, ICALP 2015, Lecture Notes in Computer Science, Vol. 9134, Springer, Berlin, 2015, pp. 605-616.

[12] L. Georgiadis, G. F. Italiano, L. Laura, N. Parotsidis, 2-vertex connectivity in directed graphs. Inf. Comput. 261 (2018) $248-264$.

[13] L. Georgiadis, G. F. Italiano, A. Karanasiou, N. Parotsidis, N. Paudel, Computing 2-connected components and maximal 2-connected subgraphs in directed graphs: An experimental study, Proceedings of the Meeting on Algorithm Engineering and Experiments (ALENEX), 2018, pp. 169-183.

[14] M. Henzinger, S. Krinninger, V. Loitzenbauer, Finding 2-edge and 2-vertex strongly connected components in quadratic time, In: Halldórsson, K. Iwama, N. Kobayashi, B. Speckmann (Eds.), Automata, Languages, and Programming, ICALP 2015, Lecture Notes in Computer Science, Vol. 9134, Springer, Berlin, 2015, pp. 713-724.

[15] G. F. Italiano, L. Laura, F. Santaroni, Finding strong bridges and strong articulation points in linear time, Theoret. Comput. Sci. 447 (2012) $74-84$.

[16] R. Jaberi, On computing the 2-vertex-connected components of directed graphs, Discrete Appl. Math. 204 (2016) $164-172$.

[17] R. Jaberi, On computing the 2-vertex-connected components of directed graphs, (2014), arXiv:1401.6000 [cs.DS].

[18] R. Jaberi, Computing the 2-blocks of directed graphs, RAIRO-Theor. Inf. Appl. 49 (2015) 93-119.

[19] R. Jaberi, Twinless articulation points and some related problems, (2019), arXiv:1912.11799 [cs.DS].

[20] W. D. Luigi, L. Georgiadis, G. F. Italiano, L. Laura, N. Parotsidis, 2-Connectivity in directed graphs: An experimental study, Proceedings of the Meeting on Algorithm Engineering and Experiments (ALENEX), 2015, pp. 173-187.

[21] T. Lengauer, R. E. Tarjan, A fast algorithm for finding dominators in a flowgraph, ACM Trans. Program. Lang. Syst. 1 (1979) $121-141$.

[22] S. Raghavan, Twinless strongly connected components, In: F. B. Alt, M. C. Fu, B. L. Golden (Eds.), Perspectives in Operations Research, Operations Research/Computer Science Interfaces Series, Vol. 36, Springer, Boston, 2006, pp. 285-304.

[23] D. J. Rose, R. E. Tarjan, Algorithmic aspects of vertex elimination, Proceedings of the Seventh Annual ACM Symposium on Theory of Computing (STOC 75), Association for Computing Machinery, New York, 1975, pp. 245-254.

[24] J. Schmidt, A simple test on 2-vertex- and 2-edge-connectivity, Inform. Process. Lett. 113 (2013) 241-244.

[25] R. E. Tarjan, Depth-first search and linear graph algorithms, SIAM J. Comput. 1 (1972) 146-160. 